Pteridines

Vol. 21, 2009, pp. $22-28$

\title{
Experiences with the SELDI-TOF Method for the Detection of the Diabetes Associated Antigen GAD 65 in the Peripheral Blood of Patients with Diabetes and Inflammation
}

\author{
Gernot P. Tilz 1 , Marco Wiltgen², Albin Hermetter ${ }^{3}$, Hannes Schmiedinger ${ }^{3}$, Ulrike Demel ${ }^{4}$ \\ ${ }^{1}$ Medical University of Graz, Graz, Austria, ${ }^{2}$ Institute for Medical Informatics, Statistics and Documentation, \\ Medical University of Graz, Graz, Austria, ${ }^{3}$ Technical University of Graz, Graz, Austria, ${ }^{4}$ Clinical Immunology, \\ Medical University of Graz, Graz, Austria
}

Date received: 2009/11/09

\begin{abstract}
The treatment of diabetes mellitus type 1 is a major challenge in health care. The diabetes associated antigen Glutamic Acid Decarboxylase (GAD 65) has been described as strictly intracellular. However an intracellular antigen would never have access to the immune system. In a previous study with the high sensitive fluorescence correlation spectroscopy (FCS), we detected the GAD 65 antigen in the sera of patients with type 1 diabetes and related autoimmune diseases. FCS is a time consuming measurement procedure and very sensitive to disturbance, which makes unsuitable for a routine screening of the patient sera for GAD 65. Since the measured GAD 65 concentrations were surprisingly high $(2.65 \mathrm{microgram} / \mathrm{ml})$ and therefore within the detection range of less sensitive methods, in this study we used the SELDI-TOF (Surface enhanced laser desorption ionisation - time of flight) technique to confirm the FCS results in view of an early diabetes diagnosis. For this purpose we developed an array with the SELDI-TOF method and analysed 4 patient sera (2 diabetes and 2 controls) in a pilot experiment. For one diabetes serum, we found some evidence for the diabetic antigen GAD 65 in the mass spectra in a peak close to albumin with a molecular weight of $65 \mathrm{kDa}$. The 3 other samples remained negative. Because of the higher concentration of albumin in the sera, a peak evaluation in regard of GAD 65 was difficult and not reliable in this setup. Troubles and experiences with SELDI-TOF for the detection of GAD 65 are discussed.
\end{abstract}

Key words: Diabetes Mellitus Type 1, autoimmunity, Glutamic Acid Decarboxylase (GAD 65) antigen, Surface enhanced laser desorption ionisation - time of flight (SELDI-TOF)

\section{Introduction}

Diabetes mellitus type 1 is an autoimmune disease with various antigen systems involved. It is one of the most debilitating and life threatening autoimmune diseases, where an early diagnosis is desirable and is also the prerequisite for successful prevention and therapy (1-5). Several attempts have been made based on genetic and metabolic analysis (6-10). In patients with critical limb ischemia inflammatory markers like tumor necrosis factor- and monocyte/macrophage product neopterin were described to be elevated in patients with diabetes as compared to non-diabetic (11). Moreover, raised urinary neopterin levels were found in islet cell antibodies (ICA) positive and 10 ICA negative diabetics at onset of disease compared with age-matched control subjects (12). In longer standing diabetics, neopterin levels were significantly higher in ICA positive than in ICA negative patients.

Glutamic Acid Decarboxylase (GAD 65) is one of the antigens playing a considerable role in diabetes mellitus type 1 and the Stiffman-syndrome $(13,14)$. However, it is located in the cellular compartment of islet-cells and it has never been shown to exist in the peripheral blood. Research on the circulating GAD 65 antigen has been neglected and its existence in the peripheral blood denied. But such a strictly intra-cytoplasmatic antigen would never have access to the immune system. In a previous publication, we described how we designed the ultrasensitive FCS (fluorescence correlation spectroscopy) technology and found GAD 65 to exist in peripheral blood as

Correspondence to: G. P. Tilz, MD, Medical University of Graz, Auenbruggerplatz 15, A-8036 Graz, Austria, gernot.tilz@meduni-graz.at 
opposed to the current opinion in the literature (15). The results of the high sensitive FCS method showed, on one hand, that the concentration of GAD 65 in sera was surprisingly high and, on the other hand, the method was very sensitive to influence by other components (other proteins, free dyes etc.) of the human serum.

Therefore, in order to have additional information about the occurrence of GAD 65 in the blood, we used a less complicated and less sensitive method apart from FCS, namely the SELDI-TOF "Surface enhanced laser desorption ionisation - time of flight" -technology. This is well established for the research on biomarkers having the advantage that no additional detection systems such as radioactivity, enzyme- or fluorochrome labelling must be employed (FCS requires labelling with fluorochromes). SELDI-TOF is a method of mass spectroscopy, where specific proteins are identified by characteristic peaks of the mass spectrogram. With the advantages of the SELDI-technique in mind we tried to confirm our previous results and to find further evidence on the existence of GAD 65 in human blood.

\section{Materials and Methods}

\section{Specimens}

Sera from 4 patients have been investigated. The sera from 2 paediatric patients with early onset of diabetes mellitus type 1 and 2 controls were kindly provided from the serum bank of the Department of Paediatrics of the Medical University of Graz. Controls consisted of persons from normal admissions to the clinic.

\section{Reagents and protein chips}

The sera were tested on a PS20 slide (by following a previously defined protocol), provided by Phadia (former Pharmacia), Sweden-diagnostics, FreiburgBreisgau, Germany. PS20 ProteinChip Arrays are preactivated with carbonyl dimidazole chemistry that covalently binds to free primary amine groups. The arrays are 8 spot chips with $2 \mathrm{~mm}$ diameter spots, spatially compatible with one column of a standard 96well microplate (9 $\mathrm{mm}$ well pitch). The monoclonal GAD 65 antibodies Z1 and Z2 (provided by Phadia) were used and fixed covalently on the PS20 protein chip. Antibody Z1 and antibody Z2 are reacting with different epitopes of the GAD 65 molecule. In a first experiment, sera without dilution were used and in a second experiment, the sera were diluted 1:20. The investigations were made with the Ciphergen system (Ciphergen Protein Chip Software 3.0) and the calibrations of the spectra were realized in point of view of the GAD 65. The Ciphergen's ProteinChip ${ }^{\circledR}$ System is comprised of a ProteinChip Reader integrated with ProteinChip Software and a personal computer to analyze proteins captured on ProteinChip Arrays.

\section{SELDI-TOF}

Mass spectroscopy was carried out according to the SELDI-TOF (Surface enhanced laser desorption ionisation - time of flight) system $(16,17)$. SELDI-TOF has been proven as suitable tool in the clinical laboratory for the profiling of biomarkers in complex biological specimens such as: serum, plasma, intestinal fluid and urine (18-26). It consists of an aluminium carrier, called the array, a laser unit and a system which measures the time of flight (TOF) of the molecules. The TOF system includes an electric field, induced by the acceleration potential, and a detector. Photomultipliers, avalanche diodes etc. are used as detectors.

Chip array: The different chip array types have either: surfaces that bind many different proteins or surfaces with a specific biomolecular affinity. The chips of the second type are composed of biochemically active surfaces, such as: immobilized antibodies or receptor proteins, and bind only specific molecules. Therefore, they are used to exploit specific molecular recognition mechanisms, enabling the focus on the proteins of interest. After putting the sample on the chip, the weakly bound molecules are washed away. The remaining sample molecules are mixed with small photosensitive molecules. These molecules cause the sample to crystallize, form the matrix as it dries and facilitate the ionization of the proteins.

Laser desorption and ionization: The chip with the samples is put into a vacuum chamber, the flight tube of the mass spectrometer. The laser pulse excites the photosensitive matrix molecules. The energy of the excited molecules is converted into thermal energy which heats up the sample spot. The overheated part of the sample mix explodes into a plume and the molecules are liberated from the array. The protein molecules in the plume collide with the excited matrix molecules, whereby the matrix molecules transfer protons to the proteins and create charged proteins. Due to repeated processes, the proteins can get multiple charges.

Mass spectrum: The counted totals per time interval are displayed in the spectrum. The detection of molecules with the same molecular weight and the same electric charge produce a signal which is called a singleton peak. A peak in the spectrum is then the signal induced by neighbouring singleton peaks. The peak 
PS 20

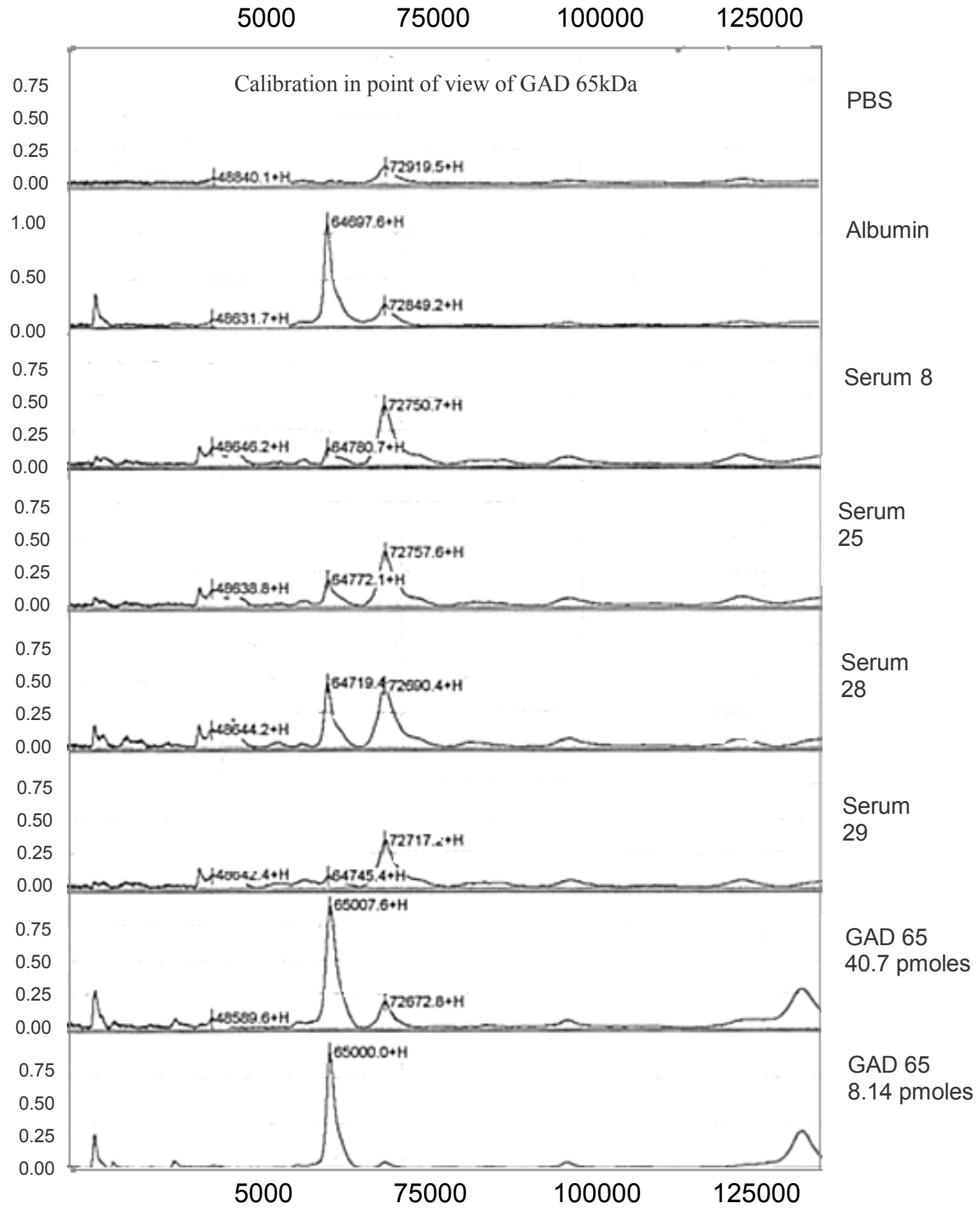

Figure 1. The spectra of the pure patient sera show very exposed peaks resulting from albumin at $64.697 \mathrm{kDa}$. The peaks at 50, 75 and $150 \mathrm{kDa}$ result from the antibodies. Because the expected GAD molecule has a molecular weight near albumin $(65 \mathrm{kDa})$, identification in patient sera under these experimental conditions is not possible. 
area is proportional to the number of detected molecules. The baseline of in the spectrum is formed by the dark current inside the detector and the detected air molecules.

\section{Results}

To realize a diagnostic procedure, every serum was tested in duplicate with different successive dilutions (for example: 1:5, 1:10). We found evidence for GAD 65 in one serum sample in the mass spectrum, which gives us a peak very close to albumin with a molecular weight of $65 \mathrm{kDa}$. This serum was from a child with an early onset of diabetes mellitus type 1 . The concentration of albumin in sera is of course tremendously higher than the concentration of the expected GAD 65. By use of the protein chip PS20 with fixation by covalent bonds of the GAD 65 antibodies, the contamination of the exuberant albumin is less disturbing. Peak evaluation after spiking experiments was done to have comparable results as with FCS (15). The spectra always show the peaks of the antibodies at 50, 75 and 150 $\mathrm{kDa}$.

Evaluation of the spectra resulting from pure sera's on the P20 slides

The spectra of the pure patient sera on the P20 slide display very exposed peaks resulting from albumin (Fig. 1). As reference, albumin was measured showing a peak at $64.697 \mathrm{kDa}$ in the spectrum. Because the expected GAD 65 molecule has a molecular weight of $65 \mathrm{kDa}$, an interpretation of the spectra of the sera under these experimental conditions is not possible.

\section{Evaluation of the spectra resulting from sera diluted at 1:20}

The spectra of the diluted sera (Fig. 2) show a much diminished peak of the reference albumin. For the interpretation of the results, we have calibrated in patient sera the obtained peak intensity at $65 \mathrm{kDa}$ in proportion to the intensity of the albumin peak in the reference. The intensity of the appropriate peak in the spectrum is greater than the peak obtained for albumin by a factor of 8.4 (Table 1 ).

\section{Discussion}

Whereas the current opinion in the literature conforms to the strict intracellular localization of GAD 65 , this hypothesis can not explain the immunization and the occurrence of GAD 65 antibodies. Our assumption was that it has to be in the serum of patients and should be found in higher concentrations before the onset of the disease, since GAD 65 has to prime the immune system as an initial step for the following pathology. Biomarkers have been proven to play an important role for the early diagnosis of diseases, such as: cancer, Alzheimer's disease etc. (27-33). Assuming that GAD 65 occurs at the early stage of diabetes mellitus type 1 and remains present during the course of the disease, it would be a suitable candidate as a biomarker.

Therefore, we started our search for the GAD 65antigen in a soluble form in the peripheral blood. Unknown concentrations let us use the ultrasensitive FCS with the astonishing result of relatively high GAD 65 concentrations (15). FCS is a very sensitive method and is even able to detect single molecules (in "clean systems"), but the technique requires a labelling of the antibodies, which is very difficult to achieve and time consuming. Furthermore, the method is very sensitive to disturbances resulting from other molecules and free dye contamination. In view of a routine diagnosis and starting experiments on a possible biomarker profiling, this time we tried a simpler method where sophisticated labelling techniques can be avoided. SELDI-TOF offered us the simplicity and the already known access to discover biomarkers in the peripheral blood in microgram amounts. This technique has the advantage that no labelling of the antibodies is necessary for the detection of the molecules. Only their molecular weight characterized by the TOF leads to the identification of the expected antigen GAD 65.

By the use of SELDI-TOF and the study of some specimens used in the previous work with FCS we found some evidence for the diabetic antigen GAD 65 in a peak close to albumin. We were successful in fixing GAD 65 in our experiments in a sandwich on the aluminium array. The albumin, produced in the liver, is the most abundant protein in human blood plasma. The reference range for albumin in blood is 35 to 53

Table 1. The intensity of the peak at $65 \mathrm{kDa}$ for specimen 28 is $8.40 \mathrm{x}$ the intensity of the corresponding peak for albumin. This serum results from a child with diabetes mellitus type 1 at an early stage.

$\begin{array}{lcccc}\text { Specimen } & \mathbf{8} & \mathbf{2 5} & \mathbf{2 8} & \mathbf{2 9} \\ \text { GAD/Albumin } & 0.95 & 1.70 & 8.40 & 2.26 \\ \text { Interpretation } & \text { negative } & \text { negative } & \text { positive } & \text { negative }\end{array}$


PS 20

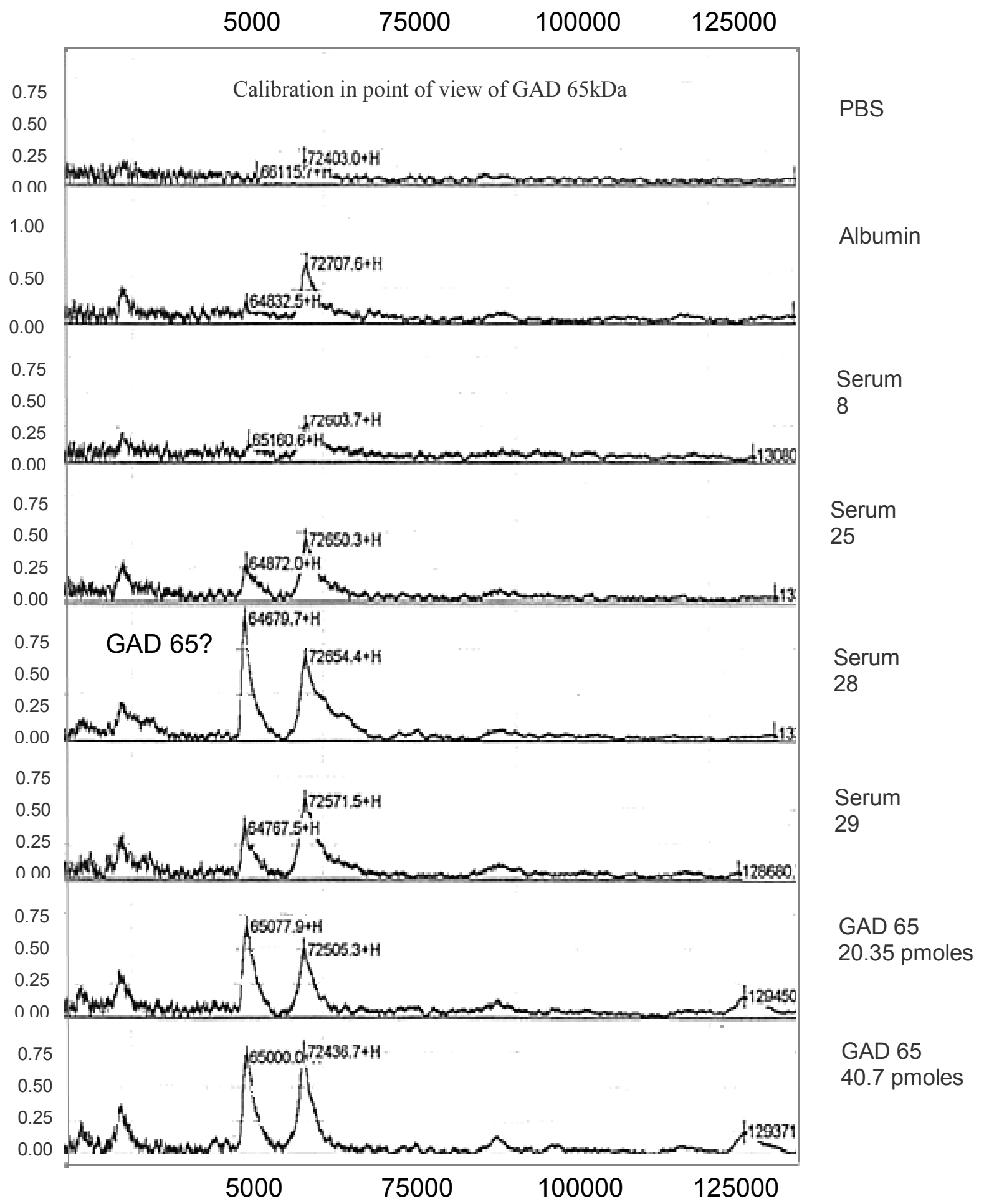

Figure 2. The sera are diluted at 1:20. The spectrum of the diluted serum 28 shows a peak at $65 \mathrm{kDa}$ which may results from GAD65. The intensity of the peak is greater, by a factor of 8.4 , than the corresponding albumin peak. This serum is from a paediatric patient with diabetes mellitus type 1 at an early stage. 
$\mathrm{mg} / \mathrm{ml}$. Its concentration is therefore higher, by a factor above 10.000, than the expected value of GAD 65 (2.65 microgram $/ \mathrm{ml}$ ). The molecular weight of albu$\min$ is $66.47 \mathrm{kDa}$ and therefore near the molecular weight of Glutamic Acid Decarboxylase $(65 \mathrm{kDa})$. The weights differ only by $2.26 \%$. Because of the tremendously higher concentration of albumin in the sera, a peak evaluation in regard of GAD 65 could not be achieved in a proper manner. Although an array with GAD 65 antibodies was used to pick out the antigen of the sera, it seems that the omnipresent albumin could not be completely washed out, but remained partially on the chip bounded by van der Waals interactions. This leads to a superposition of the GAD 65 peak and the albumin peak at approximatly $65 \mathrm{kD}$, which complicate the proper detection of the antigen. The results of these first trials show that the application of SELDITOF for the detection of GAD 65 in human sera, in the manner presented here is not reliable for a routine procedure.

In conclusion, the development of new techniques for the separation of GAD 65 from albumin is necessary for an accurate and reliable diction of the diabetes type 1 antigen. It would be also interesting to find out whether any association might exist between the appearance of GAD 65 in human blood and increased neopterin concentrations which appeared to be a sensitive indicator for the activation of cell-mediated immunity in type 1 diabetes even when ICA were undetectable (12). To obtain further evidence on the existence of GAD 65 in human blood, we used the ELISA (Enzyme-Linked Immunoabsorbent Assay) technique in a further study for the confirmation of the presence of the antigen (manuscript in preparation).

\section{Acknowledgement}

We wish to thank Ms. G. Searle for the critical reading of the text.

\section{References}

1 Hill NR, Thompson B, Bruce J, Matthews DR, Hindmarsh P. Glycaemic risk assessment in children and young people with Type 1 diabetes mellitus. Diabet Med 2009;26(7):740-3.

2 Moltchanova EV, Schreier N, Lammi N, Karvonen M. Seasonal variation of diagnosis of Type 1 diabetes mellitus in children worldwide. Diabet Med 2009; 26(7):673-8.

3 Karagianni P, Sampanis Ch, Katsoulis Ch, Miserlis G, Polyzos S, Zografou I, et al. Continuous subcutaneous insulin infusion versus multiple daily injections. Hippokratia 2009;13(2):93-6.

4 Grossi SA, Lottenberg SA, Lottenberg AM, Della Manna T, Kuperman H. Home blood glucose monitoring in type 1 diabetes mellitus. Rev Lat Am Enfermagem 2009;17(2):194-200.

5 Veleminsky $\mathrm{Sr}$ M, Buresova G. Health Related Quality of Life of Children and Adolescents with Type 1 Diabetes. Neuro Endocrinol Lett 2008;5;29(6).

6 Chatzigeorgiou A, Halapas A, Kalafatakis K, Kamper E. The use of animal models in the study of diabetes mellitus. In Vivo 2009; 23(2):245-58.

7 Bantle JP. Dietary fructose and metabolic syndrome and diabetes. J Nutr 2009;139(6):1263-8.

8 Philips JC, Marchand M, Scheen AJ. Pulse pressure and cardiovascular autonomic neuropathy according to duration of type 1 diabetes. Diabetes Metab Res Rev 2009;25(5):442-51.

9 Moltchanova EV, Schreier N, Lammi N, Karvonen M. Seasonal variation of diagnosis of Type 1 diabetes mellitus in children worldwide. Diabet Med 2009;26(7):673-8.

10 Grossi SA, Lottenberg SA, Lottenberg AM, Della Manna T, Kuperman H. Home blood glucose monitoring in type 1 diabetes mellitus. Rev Lat Am Enfermagem 2009;17(2):194-200.

11 Bertz L, Barani J, Gottsäter A, Nilsson PM, Mattiasson I, Lindblad B. Are there differences of inflammatory bio-markers between diabetic and non-diabetic patients with critical limb ischemia? Int Angiol. 2006 Dec;25(4):370-7.

12 Manna R, Salvatore M, Di Leo MA, Scuderi F, Greco AV, Ghirlanda G, et al. Relationship between urinary neopterin excretion and islet cell antibodies in type 1 (insulin-dependent) diabetes. Diabetes Res 1991;17(1):33-6.

13 Franke B, Galloway TS, Wilkin TJ. Developments in the prediction of type 1 diabetes mellitus, with special reference to insulin autoantibodies. Diabetes Metab Res Rev. 2005;21(5):395-415.

14 Harrison LC. Islet cell antigens in insulin-dependent diabetes: Pandora's box revisited. Immunol Today 1992;13(9):348-52.

15 Tilz GP, Dausset J, Wiltgen M. The diabetic antigen glutamic acid decarboxylase (GAD 65) in the human peripheral blood. Int Arch Allergy Immunol 2009;152(2):184-94.

16 Dijkstra M, Vonk RJ, Jansen RC. SELDI-TOF mass spectra: A view on sources of variation. $\mathrm{J}$ Chromatography B 2007;847:12-23.

17 Issaq HJ, Conrads TP, Prieto DA, Tirumalai R, Veenstra TD. SELDI-TOF MS for diagnostic proteomics. Anal Chem A 2003;75(7):148A-55A.

18 Liu J, Zheng S, Yu J, Zhang J, Chen Z. Serum pro- 
tein fingerprinting coupled with artificial network distinguishes glioma from healthy population or brain benign tumour. J Zhjiang Univ SCI 2004; 6B, 1:4-10.

19 Clarke CH, Buckley JA, Fung ET. SELDI-TOFMS proteomics of breast cancer. Clin Chem Lab Med 2005;43;12:1314-20.

20 Boyce EA, Kohn EC. Ovarian cancer in the proteomics era: diagnosis, prognosis and therapeutics targets. Int J Gynecol cancer 2005; 15: 266-373.

21 Li J, Orlandi R, White CN, Rosenzweig J, Zhao J, Sergni E, Morelli D, et al. Independent Validation of Candidate Breast Cancer Serum Biomarkers Identified by Mass spectrometry. Clinical Chemistry 2005; 51, 12: 2229-2235.

22 Fung ET, Yip T, Lomas L, Wang Z, Yip C, Meng X, et al. Classification of cancer types by measuring variants of host response proteins using SELDI serum assays. Int J cancer 2005;115:783-9.

23 Vorderwülbecke S, Cleverley S, Weinberger SR, Wiesner A. Protein quantification by the SELDITOF-MS-based ProteinChip $®$ System. Nature Methods 2005;2(5):393-5.

24 Tomosugi N, Kitagawa K, Takahashi N, Sugai S, Ishikawa I. Diagnostic Potential of tear Proteomic Patterns in Sjögren's Syndrome. Journal of Proteome Research 2005;4:820-5.

25 Gast MC, Van Gils CH, Wessels LF, Harris N, Bonfrer JM, Rutgers EJ, et al. Serum protein profiling for diagnosis of breast cancer using SELDITOF MS.Oncol Rep 2009;22(1):205-13.

26 Lebrecht A, Boehm D, Schmidt M, Koelbl H, Grus FH. Surface-enhanced laser desorption/ionisation time-of-flight mass spectrometry to detect breast cancer markers in tears and serum. Cancer Genom Proteom 2009;6(2):75-83.
27 Ikeda N, Nagase S, Ohira T. Individualized adjuvant chemotherapy for surgically resected lung cancer and the roles of biomarkers. Ann Thorac Cardiovasc Surg 2009;15(3):144-9.

28 Hoimes CJ, Moyer MT, Saif MW. Biomarkers for early detection and screening in pancreatic cancer. Highlights from the 45th ASCO annual meeting. Orlando, FL, USA. May 29-June 2, 2009. JOP 2009;6:10(4):352-6.

29 Horváth I, Lázár Z, Gyulai N, Kollai M, Losonczy G. Exhaled biomarkers in lung cancer. Eur Respir J 2009;34(1):261-75.

30 Walther A, Johnstone E, Swanton C, Midgley R, Tomlinson I, Kerr D. Genetic prognostic and predictive markers in colorectal cancer. Nat Rev Cancer 2009;9(7):489-99.

31 Loussouarn D, Campion L, Leclair F, Campone M, Charbonnel C, Ricolleau G, et al. Validation of UBE2C protein as a prognostic marker in nodepositive breast cancer. $\mathrm{Br} \mathrm{J}$ Cancer 2009; 101(1):166-73.

32 Hampel H, Broich K, Hoessler Y, Pantel J. Biological markers for early detection and pharmacological treatment of Alzheimer's disease. Dialogues Clin Neurosci 2009;11(2):141-57.

33 Petrie EC, Cross DJ, Galasko D, Schellenberg GD, Raskind MA, Peskind ER, et al. Preclinical evidence of Alzheimer changes: convergent cerebrospinal fluid biomarker and fluorodeoxyglucose positron emission tomography findings. Arch Neurol 2009;66(5):632-7. 